\title{
2-ion and 3-ion neighbor spatial distribution functions in plasmas
}

\author{
D.V. Fisher ${ }^{* 1,2}$ and Y. Maron ${ }^{1}$ \\ ${ }^{1}$ Faculty of Physics, Weizmann Institute of Science, Rehovot 76100, Israel \\ 2 present primary address: Soreq NRC, Yavneh 81800 , Israel
}

Received 4 May 2003, accepted 31 July 2003

Published online 10 October 2003

Key words Electron state collectivization, tunneling, quasimolecule

PACS 52.27.Gr,61.20.Ne,52.65.Yy

\begin{abstract}
Accurate treatment of the plasma density effects requires a detailed knowledge of spatial distribution of individual ions around a test ion. The 2-body and 3-body distribution functions involving the nearest neighbor $(\mathrm{NN})$ and the next-nearest neighbor (NNN) ions have recently been derived [1], valid for both ideal and nonideal plasmas. Here we apply these results to determine the effect of a few individual surrounding ions on the dense plasma electron states and, in particular, on the electron state collectivization by tunneling. Validity of the transient diatomic quasimolecule model, found in literature, is discussed.
\end{abstract}

The principal challenge in the research of plasmas and liquids is brought along by the absence of a long-range order. Microconditions at locations of individual ions differ substantially even in a macroscopically uniform sample. Determination of local spatial distribution of particles surrounding any given ion in plasma ("test ion") is thus one of the fundamental problems of plasma physics. Knowledge of this distribution is important for treatment of electron states, modeling of plasma composition, determination of radiative and transport properties, equations of state, phase transitions, critical phenomena, and fusion.

The local microfield distribution in plasmas is being investigated for over 80 years [2]. However, for majority of the problems concerning nonideal plasmas the uniformity of a local microfield can not be assumed [2,3], and the detailed knowledge of potential around a given ion location is required. Towards this end, many-body spatial distribution functions involving the test ion and one, two, and ultimately more of its near neighbor ions must be determined.

Surprisingly, the near-neighbor spatial distribution functions have not been extensively studied until recently, although a related object, a pair distribution function $g(r)$, has been studied in detail for several decades [4]. The 2-body NN distribution function $P_{N N}(r)$ describes the probability to find the NN ion at the distance $r$ from a given ion (which implies the absence of other ions closer than $r$ to the given ion). By contrast, a pair distribution function $g(r)$ describes the probability to find an ion at the distance $r$ from a given ion regardless of the fact that there may be other ion(s) located closer to the given ion. For that reason, $g(r)$ is not well suited for the determination of the 2-body and 3-body near-neighbor distribution functions (NNDF).

We have found out [1] that the most convenient way to determine the NNDF is to express them as functionals of the local potentials. The expression obtained for the 2-body distribution function

$$
P_{N N}(r)=\frac{3 r^{2}}{a^{3}} \exp \left\{-\frac{Z_{i} e}{T} U_{N N}(r)-\int_{0}^{r} \frac{3 s^{2} d s}{a^{3}} \exp \left[-\frac{Z_{i} e}{T} U_{N N}(s)\right]\right\}
$$

is exact in equilibrium. Here $a=\left(4 \pi n_{i} / 3\right)^{-1 / 3}$ is the typical interionic distance, and $U_{N N}(r)$ is an effective local potential at the distance $r$ from the test ion provided there are no other ions within $r$. The expression for $U_{N N}(r)$ is not apriori known, and must be determined semi-empirically. In particular, for plasmas $U_{N N}(r)$ includes contributions from the test ion itself and from the free electrons within $r$ from the test ion. The approximate expression for the 3-body distribution function is given in Ref. [1], along with a recipe for approximate

\footnotetext{
* Corresponding author: e-mail: fndima@ plasma-gate.weizmann.ac.il
} 
determination of N-body NNDF for $N>3$. The analytic expressions obtained for 2-body and 3-body NNDF agree very well with the results of molecular dynamics (MD) simulations, see Ref. [1]. The MD simulations were carried out accounting for Coulomb interactions between all particles, and did not utilize $U_{N N}(r)$.

The near-neighbor effects on ions in plasmas manifest themselves, in particular, via energy level shift and broadening (Stark effect), electron state collectivization, and screening. Screening effect is produced not by the near neighbors alone, but rather by all particles inside the screening sphere, collectively. By contrast, electron state collectivization is governed by the effect of a few near-neighbor ions. Collectivized state is a negativeenergy state bound in several (rather than one) ionic potential wells. Electron state is collectivized when an electron can move between the potential wells, either above the potential barrier peak or by tunneling, with no inelastic process being involved [3]. For a proper description of collectivization conditions it is important to know the distribution $P_{\text {rat }}(\zeta)$ of the ratio $\zeta=\rho / r$, where $\rho$ is the distance to NNN ion. It is easy to show that in both ideal and nonideal plasmas $P_{\text {rat }}(\zeta)$ decays fast for $\zeta>1$. In the ideal plasmas the decay obeys a power law while in nonideal plasmas (no matter how weakly nonideal) at large $\zeta$ values the decay is exponential [1].

In order to provide an amenable description of the many-body effects on the ions in plasmas, it is crucial to determine how many near-neighbor ions must be considered in order to reproduce adequately the ionic potential in the vicinity of the test ion. "Vicinity" here is a sphere with the radius matching the characteristic size of the testion optical electron wavefunction. In weakly-coupled plasmas this radius, for most electron states considered, is notably smaller than $a$. Hence, local microfield in weakly-coupled plasmas can be assumed uniform, except for the case of a close ion-ion collision. In nonideal plasmas, on the other hand, even the low-lying excited states of the dominant ionization stages have wavefunction radii comparable to $a / 2$. These states become substantially affected by the effect of neighbor ions. For ion-ion coupling parameter $\Gamma=\left(Z_{i} e\right)^{2} / a T>1$ this becomes an issue even for the ground states of ions. It is then important to determine how many near-neighbor ions must be taken into account in order to describe amenably the effect on the wavefunctions.

In literature, it has been suggested that in order to provide an accurate interpretation and modeling of dense plasma spectral line shapes (and, possibly, ionization-stage composition), a system including an ion and its $\mathrm{NN}$ ion can be viewed as a transient diatomic quasimolecule (TDQM) [5, 6]. That is to suggest, it is adequate to account for an effect of the NN ion alone on the wavefunction of the test-ion optical electron. The argument is still ongoing as to whether the TDQM-specific features can be observed in dense plasma spectra.

We contend that the picture of ions in nonideal plasma forming TDQMs is substantially oversimplified. The rapid decrease of $P_{\text {rat }}(\zeta)$ distribution function at $\zeta>1$ suggests that the distance to the NNN ion is seldom significantly larger than the distance to the $\mathrm{NN}$ ion even in ideal plasmas, and even more seldom in the nonideal plasmas (unless the full ion-ion interaction potential becomes attractive and true molecules are formed). Thus, in plasmas where the dominant contribution to the ion-ion interaction potential at characteristic interatomic distances is the Coulomb repulsion, for any ion there are several neighbor ions located at approximately the same distance from that ion, and therefore taking the effect of only one of them and disregarding the others seems unjustified. To find out what the actual number of neighbor ions is for a test ion in plasma, we introduce the following two quantities: the close neighbor fraction $C_{1}$ and the effective coordination number $K_{\text {eff }}$. The quantity $C_{1}$ (which is a function of macroscopic plasma parameters) describes the probability that a given ion is its $\mathrm{NN}$ ion's NN. Indeed, when an ion B is the NN of an ion A it is not at all necessary that an ion A is the NN of an ion $\mathrm{B}$. We also introduce a complementary quantity $C_{0}=1-C_{1}$. For perfect lattices, the coordination number $K$ is the number of ions located closest to a given ion. Here, we generalize this definition utilizing the value of $C_{1}$. For crystal lattices above Debye temperature but below melting temperature, the displacements of individual ions from their average positions in the lattice may be assumed to be small (compared to the lattice constant), uncorrelated with each-other, and gaussian. Under these conditions, $C_{1}$ value depends only on the lattice type, and does not depend on thermodynamic parameters. Of course, the larger is the lattice coordination numbers the larger is $C_{0}$, as there is a larger number of near neighbors to choose the NN ion from. For most Bravais lattices $C_{0} \approx 0.4-0.5$. Remarkably, for ideal plasma $C_{0_{i d}}=0.406$, close to the value $C_{0_{s c}}=0.414$ of a simple cubic lattice $(K=6)$, and larger than the $C_{0_{s t}}=0.373$ value of a simple tetragonal lattice with $K=4$. This suggests that the $K_{\text {eff }}$ value for an ideal plasma is between 5 and 6 . By contrast, if the TDQM approximation was correct and ideal-plasma $K_{\text {eff }}$ was close to 1 , one would have obtained ideal-plasma $C_{0}$ value close to zero (indeed, $C_{0}=0$ for $K=1$ ). In nonideal plasmas, with an increase in a coupling constant (by changing the plasma parameters along some thermodynamic path), $C_{0}$ value increases somewhat, and approaches smoothly its 
lattice value as plasma crystallizes, provided that along that path the ion-ion interaction at characteristic interionic distance remains repulsive.

Following example illustrates the importance of an account for more than one near-neighbor ion. Consider tunneling of an electron between its parent ion potential well and the potential well of the NN ion in dense plasma. If the tunneling occurs on a sufficiently short time-scale, such an electron state is deemed collectivized [3]. To determine the tunneling probability per unit time it is crucial to account for the perturbation produced by the spectator ions, i.e., near neighbor ions other than the NN ion. Potential of the parent ion and the $\mathrm{NN}$ ion is an axially-symmetric two-well potential (2WP). The spectator ion contribution to the potential i) breaks the axial symmetry of the $2 \mathrm{WP}$ and ii) raises or lowers the potential barrier peak with respect to the tunneling electron energy level. Both of these contributions affect the tunneling probability substantially. Let us consider the first one here. In 2WP a direction of microfield at the test ion location is collinear with the tunneling path. We assume the Stark effect to be linear. Direction of the local microfield at the test ion location governs then the splitting of the energy levels into Stark components. In 2WP the z-axis for the Stark components coincides with the tunneling path. For Stark components with large z-projection of angular momentum the tunneling probability is strongly reduced, see e.g. [7,8]. When spectator ion contribution to the potential is taken into account, the situation turns out to be quite different. Figure 1 shows a typical potential in yz-plane, in presence of a single spectator ion. As one can see, the tunneling path remains close to the original z-axis, however, now the microfield at the parent ion location is not collinear with the tunneling path. Consequently, angular-momentum projection on the tunneling path is not conserved, and the aforementioned reduction in tunneling probability does not occur. Figure 2 shows

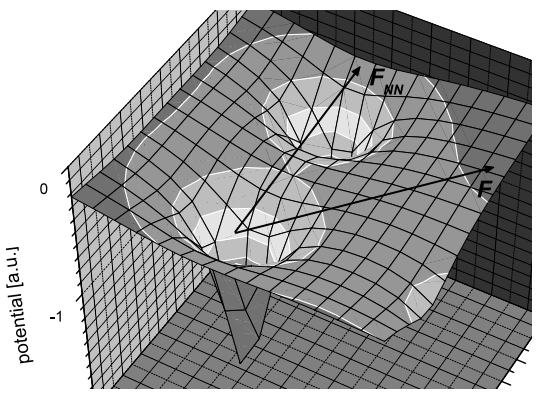

Fig. 1 Typical 3-ion potential.

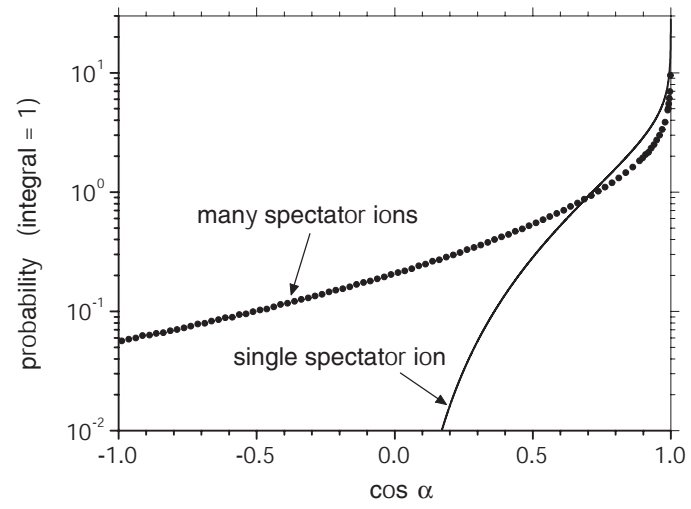

Fig. 2 distributions of $\cos \alpha$

distribution of cosine of the angle $\alpha$ between the direction of the microfield at test ion location and the direction to the NN ion in ideal plasma, for the cases of a single spectator ion and of a large number of spectator ions. In the latter case the distribution is obtained by MD simulations, while in the singe-spectator case the distribution was found analytically. Due to the $\sin \alpha \Delta \alpha$ factor, both distributions yield zero probabilities to find the total field collinear with the direction to the NN ion ( $\alpha=0$ ). Specifically, the probability to find $\alpha<\alpha_{0} \ll 1$ is proportional to $\alpha_{0}^{k}$ where $k>1$. In ideal plasma $k=3 / 2$ both in the single-spectator and multi-spectator cases, while in nonideal plasmas $k=2$. Details will be published separately.

\section{References}

[1] D. V. Fisher, Y. Maron, Eur. Phys. J. D 14, 349 (2001)

[2] H. R. Griem, Principles of Plasma Spectroscopy (Cambridge University Press, 1997).

[3] D. V. Fisher, Y. Maron, Eur. Phys. J. D 18, 93 (2002).

[4] I. Z. Fisher, Statistical Theory of Liquids (Chicago University Press, 1964).

[5] E. Leboucher-Dalimier, A. Poquérusse, P. Angelo, Phys. Rev. E 47, R1467 (1993); P. Gauthier, S. J. Rose, P. Sauvan, P. Angelo, E. Leboucher-Dalimier, A. Calisti, B. Talin, Phys. Rev. E 58, 942 (1998).

[6] E. Oks, Phys. Rev. Lett. 85, 2084 (2000); E. Oks, Phys. Rev. E 63, 057401 (2001).

[7] A. M. Perelomov, V. S. Popov, M. V. Terent'ev, Sov. Phys. JETP 23, 924 (1966).

[8] D. V. Fisher, Y. Maron, L. P. Pitaevskii, Phys. Rev. A 58, 2214 (1998). 